\title{
PROPUESTA DE ACCIONES PARA MEJORAR INGRESOS Y NÚMERO DE CRUCERISTAS EN LA AGENCIA VIAJES CUBANACÁN SUCURSAL OCCIDENTE.
}

\section{PROPOSAL OF ACTIONS TO IMPROVE REVENUES AND NUMBER OF CRUISE PASSENGERS IN THE TRAVEL AGENCY CUBANACÁN BRANCH WEST.}

\author{
Maité Rodriguez González, MSc. \\ Máster en Gestión Turística (Cuba). \\ Profesora y Jefe de Departamento Turismo y Viajes en la Facultad de Turismo de \\ la Universidad de La Habana, Cuba. \\ mrodriguez@ftur.uh.cu

\begin{abstract}
Manuel Ramón González Herrera, Ph.D.
Doctor en Ciencias Geográficas, especialidad en Turismo (España). Docente-Investigador y Consultor del Departamento de Ciencias Administrativas (Programa de Turismo) de la Universidad Autónoma de Ciudad Juárez, México. manglez04@yahoo.es
\end{abstract}

\section{ARTÍCULO DE INVESTIGACIÓN}

Recibido: 26 de marzo de 2019.

Aceptado: 29 de abril de 2019.

\section{RESUMEN}

El crucerismo es la modalidad turística de más rápido crecimiento, y dentro de las principales zonas donde se desarrolla, el Caribe se consolida como el destino de mayor frecuencia. Cuba presenta condiciones favorables para el desarrollo sustentable de esta actividad, donde juegan un papel fundamental las agencias de viajes y los servicios que ofrecen a compañías de cruceros y cruceristas. Precisamente, la Sucursal Occidente de la Agencia Viajes Cubanacán en la última temporada ha mantenido un bajo rendimiento tanto en la cantidad de cruceristas atendidos como en los ingresos alcanzados. Por tanto, se realizó un estudio para determinar los factores que inciden en esta situación a fin de proponer acciones para revertirla. Para ello, se aplicó un procedimiento de diagnóstico a partir de entrevistas a especialistas, encuestas a los clientes externos y la consulta a los documentos oficiales de la Sucursal, para lo que se emplearon otras herramientas como el análisis de las cinco fuerzas de Porter, el diagrama de Pareto y la matriz DAFO con impactos cruzados. Esto permitió conocer los principales factores que afectan el desempeño de la Sucursal Occidente 
de la Agencia Viajes Cubanacán, mejoras que contribuyeran a revertir la situación actual de la entidad con respecto a la atención a compañías de cruceros y cruceristas.

Palabras clave: agencia de viajes, cruceristas, mejoras, cruceros, ingresos.

\section{ABSTRACT}

Cruise activity is the fastest growing tourism modality, and within the main areas where it is developed, the Caribbean is consolidated as the most frequent destination. Cuba presents favorable conditions for the sustainable development of this activity, where travel agencies and the services offered to cruise and cruise passengers play a fundamental role. Precisely the Western Branch of the Travel agency "Cubanacán" in the last season has maintained a low performance in both the number of cruise passengers served and the revenues achieved. Therefore, a study was conducted to determine the factors that affect this situation in order to propose actions to reverse it. To do this, a diagnostic procedure was applied based on interviews with specialists, external customers and consultation of the official documents of the Branch, on which other tools were used, such as the analysis of Porter's five forces, the Pareto diagram and the SWOT matrix with crossed impacts. This allowed to know the main factors that affect the performance of the Western Branch of the Travel Agency "Cubanacán", improvements that contribute to revert the current situation of the entity with respect to the attention to cruise companies and cruise passengers.

Keywords: Travel agency, Cruise Passengers, Cruises, Income

\section{INTRODUCCIÓN}

El turismo constituye en la actualidad una actividad económica de gran relevancia a nivel internacional. El mismo tiene un carácter dinámico e integrador de diferentes sectores de la economía, especialmente al sector del transporte. La combinación sinérgica de estas dos actividades ha dado como resultado el desarrollo amplio y sostenido de una modalidad turística bien importante: el turismo de Cruceros.

Precisamente la región del Caribe cuenta con un gran posicionamiento dentro del turismo de Cruceros siendo líder mundial, tomando en cuenta que tiene los mayores índices de visitas turísticas del mundo. Según la revista Caribbean News Digital (s/a), la región caribeña recibe anualmente un promedio de 50 mil embarcaciones de todo tipo y se estima que aproximadamente el $60 \%$ de los pasajeros de crucero visitan el Caribe. 
A pesar de que Cuba es un actor significativo para el desarrollo del turismo en la región, el número de cruceristas recibidos es considerablemente inferior al de otros países del área. El mercado norteamericano, es el principal emisor dentro de esta modalidad, y aunque persisten las restricciones del bloqueo, desde el año 2016 han comenzado a operar en la Isla importantes compañías estadounidenses como son: Carnival Cruises, Norwegian Cruise Line y Royal Caribbean Cruises.

Cubanacán Viajes, es una de las principales agencias de viajes del país, siendo un receptivo con más de 20 años de presencia en el mercado. Cuenta además con el privilegio de tener contratos exclusivos con las principales líneas de cruceros de origen europeo que operan en el país, MSC Cruceros, Crystal Cruises. A pesar de ello, los ingresos y el número de cruceristas son inferiores al deseado por la agencia. Según el Reporte de Análisis de Gestión Comercial de Excursiones, realizado en noviembre de 2018, se creció con respecto al 2017 y 2016 en el número de pax y los ingresos; sin embargo, no se logró cumplir el plan acumulado para esos años.

En el análisis anterior se adjudica, en buena medida este resultado, al bajo rendimiento de las ventas en los cruceros con los que ya se tienen contratos, así como la no entrada de otros dos que estaban planificados. Por lo que se plantea como objetivo de la investigación: Proponer acciones que permitan a la Sucursal Occidente de la Agencia Viajes Cubanacán mejorar el número de cruceristas e ingresos.

\section{REVISIÓN TEÓRICA}

\section{Aproximaciones al turismo de cruceros y la gestión del mismo desde las agencias de viajes.}

El paso del tiempo y la evolución de las tecnologías han generado cambios sustanciales en el desarrollo de esta actividad, tanto en el nivel de implicación de los consumidores, como en la manera en que las empresas se relacionan contractualmente y se adaptan a las nuevas condiciones; todo lo cual ha provocado que el fenómeno sea más complejo en la actualidad. De acuerdo con la naturaleza y características de los fenómenos que se desarrollan mediante el turismo, el mismo ha quedado dividido en diversas modalidades: cultural, sol y playa, naturaleza, náutica y de salud (Ayala, 2014).

Precisamente el turismo náutico se puede entender, como un conjunto de relaciones entre personas, surgidas al efectuar un viaje y, con la característica de que la principal motivación 
es la realización de actividades náuticas, con ánimo recreativo o deportivo, y realizadas en grandes masas de agua, como el mar, los ríos y los lagos. El recurso es la instalación náutica de recreo (Mustelier, 2015).

Para Yi (2018), el turismo de cruceros, por definición, es una sub-modalidad turística incluida en el turismo náutico, si se clasifica basándose fundamentalmente en el tipo de actividad náutica que el turista realiza en su tiempo libre, dedicado a las vacaciones. Siguiendo a este autor, el crucerismo se caracteriza por el uso de grandes embarcaciones para el transporte de pasajeros. Estos pasajeros se pueden considerar turistas náuticos, ya que la navegación en el barco de una compañía de cruceros turísticos es a todos los efectos una actividad náutica, si bien se distingue por su enfoque orientado fundamentalmente al ocio y la recreación, alejado de planteamientos activos como el deportivo (Association, 2004).

Debido al gran alcance que posee el turismo de cruceros, otros autores lo clasifican como una modalidad por sí mismo. Tal es el caso de Perelló (2006) quienes definen que es un tipo de turismo con el que se obtiene un importante volumen de negocio considerando los gastos de los turistas, de la tripulación y el aprovisionamiento del buque, por lo que permite una mejor redistribución de la riqueza en la localidad que pueda llegar a acogerlo.

Dentro de este escenario mundial, un número relativamente reducido de compañías de cruceros, compiten en diferentes formas por cuotas de mercado, desarrollando estrategias comerciales innovadoras e invirtiendo presupuestos multimillonarios en la investigación y desarrollo de novedosos buques, capaces de combinar la elegancia de espacios habitables de alta calidad, con los más modernos conceptos técnicos para proporcionar estabilidad a estos nuevos hoteles flotantes.

De acuerdo con el Cruise Industry Outlook (2018), ofrecido por la Asociación Internacional de Compañías de Cruceros, el principal mercado emisor de cruceristas es Estados Unidos. En términos generales, el producto crucero proporciona altos niveles de satisfacción. La mayoría de las personas que realizan un viaje en crucero se convierten, posteriormente, en clientes reincidentes. Una tendencia actual en la demanda turística es el aumento de las compras directas, especialmente por Internet; sin embargo, la industria de cruceros se aparta de esta realidad.

Los cruceros son un producto más complejo que otros, puesto que intervienen muchas variables como los servicios ofrecidos a bordo, las distintas opciones de destino, la duración o los puertos de embarque y desembarque. Todo esto hace que su asesoramiento sea 
determinante en muchas ocasiones para que el crucerista escoja aquella opción que mejor se adecúa a sus necesidades.

La relación entre las agencias y las navieras ha sido, es y continuará siendo clave para el desarrollo de nuestro sector. Los agentes tienen un rol fundamental en este proceso, ya que sirven como consejeros de confianza a nuestros pasajeros potenciales cuando exploran la grandísima variedad de opciones disponibles para unas vacaciones en crucero.

El Caribe es el principal destino de las compañías de Cruceros. Evidencia de ello se puede constatar en el State of the Industry Annual Report (2017) del medio especializado Cruise Industry News, en los últimos cinco años el Caribe ha engrosado su cuota desde 42,7 a $43,2 \%$ del mercado mundial, el cual se ha hecho más competitivo, con buques de más tonelaje ingresando en la región caribeña. Sus puertos desarrollan una carrera para responder a esa demanda (nuevas terminales, nuevos muelles e incentivos a las navieras), potenciando factores diferenciadores, que van desde excursiones en tierra hasta los descuentos en los destinos.

A pesar de la excelente ubicación geográfica de Cuba, el país no cuenta con un desarrollo notable dentro del turismo de cruceros. Es en el año 1995 que reaparece una embarcación de este tipo en la Isla, y a partir de entonces, se fueron incorporando compañías de forma lenta pero gradual, principalmente provenientes de Europa. No es hasta el año 2016 que se incorporan las líneas de cruceros estadounidenses Norwegian Cruises Line y Carnival Cruises, dos de las compañías más grandes a escala global.

A partir de esta fecha el crecimiento de la actividad crucerista ha sido vertiginoso, lo cual ha sido un importante aporte de ingresos a la actividad turística de la nación, ya que esta industria genera cuantiosas utilidades (Yi, 2018). No obstante, la actividad de cruceros en el país podría ser mucho mayor de no ser por las restricciones del bloqueo económicocomercial y financiero impuesto a la Isla por el Gobierno de los Estados Unidos.

Con respecto a la definición de servicio, una de las más empleadas en el sector turístico cubano es la ofrecida por Ulacia (2007) quien expresa que, un servicio es la combinación de elementos tangibles e intangibles en actividades organizadas para producir una experiencia satisfactoria para el cliente, en el momento que contacta con el producto creado por la organización. 
La gestión del servicio se considera como un método de gestión empresarial donde el servicio se constituye en el motor impulsor del funcionamiento y desarrollo. Según este planteamiento se reconocen tres dimensiones fundamentales de la gerencia: la calidad del producto, la calidad del servicio y la reducción del costo (Ulacia, 2015). Por su parte Carlzon (1991), plantea un Modelo de Gestión de Servicios que establece claramente el impacto del servicio en los resultados operativos de las entidades.

La filosofía del modelo de gerencia del Servicio de Carlzon (1991) es reflejada de una forma más ilustrativa por Albrecht (1991) en el Triángulo del Servicio (TS); el cual es definido por Ulacia (2015) como instrumento fundamental para la operación y gestión de servicios. El mismo constituye, en nuestros tiempos, una de las mejores maneras de manejar el servicio, en tanto ayuda a los directivos a meditar en lo que necesitan hacer y establecer en una filosofía de gestión. En este sentido, se convierte en el modelo por excelencia de una buena gestión del servicio, al establecer el vínculo entre la empresa y el cliente, mediante una relación flexible con otros dos elementos: los sistemas de prestación de servicios y el personal.

\section{MATERIALES Y MÉTODOS}

Se emplearon fuentes de información indirectas, tanto externas como internas, referentes en el análisis documental realizado, ya fuese de bibliografía general ajustada a las particularidades de la investigación, como documentos oficiales de la Agencia Viajes Cubanacán S.A. y su Sucursal Occidente. Además, se utilizaron fuentes primarias, las que constituyeron vías certeras de validación de los resultados obtenidos, mediante encuestas y entrevistas que permitieron profundizar en el análisis y obtener satisfactorios resultados.

Se utilizaron métodos teóricos cualitativos que permitieron el acercamiento a las tendencias y actualidad del turismo de cruceros, tales como análisis-síntesis, inductivo-deductivo y el análisis histórico-lógico. Como métodos empíricos se utilizaron la observación no participativa, la entrevista de tipo semiestructurada, el análisis documental y la encuesta. Se utilizaron además algunos métodos estadísticos que permitieron procesar las encuestas aplicadas.

\section{Definición del procedimiento a utilizar.}

Se utilizó el procedimiento descrito por Díaz (2016) atendiendo a que se ajusta a los requerimientos de la presente investigación, el mismo fue modificado por el autor dado las 
características del objeto de estudio. El procedimiento se compone de cinco (5) fases con un total de cuatro (2) etapas consecutivas.

Fase 1. Caracterización general de la agencia Viajes Cubanacán Sucursal Occidente: Para ello se identificó la sucursal donde se desarrolló el diagnóstico; y se explicó su surgimiento, ubicación, estructura organizativa, objeto social, misión y visión a que respondía, servicios y productos turísticos que ofrecía al mercado, áreas básicas de gestión y departamentos en los que se distribuía.

Fase 2. Análisis externo: El análisis externo se realizó tomando como referencia los reportes en la literatura consultada, así como, el análisis documental de las estadísticas registradas por la ONEI $(2017,2018)$. El cual facilitó la determinación de oportunidades y amenazas que influyen en la situación de esta. Este análisis se dividió en dos niveles: macroentorno o entorno general y microentorno o entorno competitivo.

El estudio del macroentorno centró su atención en el conjunto de factores económicos, político-legales, tecnológicos, social-culturales y medioambientales, considerados de importancia para delimitar el escenario en el que operaba la agencia y su influencia en el accionar de la actividad de los cruceros en la misma. Por su parte en el análisis del microentorno se concretaron las cinco (5) fuerzas competitivas planteadas por Porter (1985): poder de negociación con los clientes, así como con los proveedores, amenaza de competidores potenciales, amenaza de productos sustitutivos y la rivalidad existente entre los competidores reales, enfatizando en el análisis correspondiente a la situación de los competidores y de los clientes.

Con respecto a la competencia se delimitaron los competidores potenciales y reales, especificando el competidor superior para la agencia, por lo que se caracterizaron teniendo en cuenta su fortaleza con respecto a principales servicios y productos, así como su comportamiento en un período determinado.

El análisis de los clientes estuvo basado en los resultados obtenidos a partir de las encuestas aplicadas, así como en la valoración de los mercados que tenía definido la Sucursal. Se incluyeron, además, las tendencias actuales de los mercados del destino, así como aspectos puntuales de la gestión del servicio a cruceros en la agencia de viajes.

Para la realización de este análisis se utilizó el método de la observación no participativa. Además, se contó con el apoyo de especialistas de la entidad para cuya selección se tuvo 
en cuenta que tuviesen relación con la temática a estudiar y que conocieran las particularidades de la operación en la agencia.

Posteriormente se les explicó las características de la investigación a desarrollar, sus objetivos, técnicas y métodos a emplear para ello. Las oportunidades y fortalezas fueron ordenadas tomando en consideración el nivel de impacto que pudieran tener en la gestión del servicio a cruceros en la agencia, de modo que fueran aprovechadas para contrarrestar la incidencia de las problemáticas detectadas en el análisis.

Fase 3. Análisis interno: El análisis interno permitió concretar con exactitud las principales fortalezas y debilidades que presentaban los servicios a cruceros en la agencia de viajes. Para ello se desarrollaron las etapas siguientes:

Etapa 1. Caracterización de los servicios a cruceros en la agencia de viajes: Esta etapa permitió detallar las particularidades de la distribución en la Sucursal, mediante el análisis de varios aspectos.

Los servicios a cruceros en la agencia: Se caracterizó la gestión del servicio a cruceros brindado por la agencia, para lo cual se definieron sus principales servicios y se analizó el proceso de la gestión del servicio a cruceros utilizado.

Análisis del producto, precio, comunicación y ventas: Se realizó a partir de los resultados obtenidos en las entrevistas y encuestas aplicadas a los especialistas. En el análisis del producto se hizo referencia a la cartera de productos de la agencia orientada a la actividad de los cruceros, determinando las particularidades que caracterizaban el diseño y actualización de esta. Además, basado en la información documental entregada por la agencia se estableció una correspondencia entre los principales productos vendidos y la totalidad de opcionales incluidas en la cartera, constatando el estado actual de las ofertas de la agencia y los elementos que distinguen su forma de gestión.

De igual forma, se analizaron los precios según los mercados que la agencia tiene definidos para este servicio, se analizó y caracterizó, además, el proceso de venta de los servicios a cruceros en la agencia. De igual manera se hizo una valoración general de los recursos económicos, humanos y materiales con que cuenta la agencia para desarrollar el servicio a cruceros en la agencia, tanto en la red de ventas como en la sede de la Sucursal. 
Etapa 2. Estrategias desarrolladas para la gestión del servicio a cruceros en la agencia: En esta etapa se analizó si existía una Estrategia de servicio definida en la agencia de viajes, para conocer aquellos objetivos y políticas comerciales que se enfocaban a la gestión del servicio a cruceros en la agencia de viajes.

Fase 4. Análisis y sistematización de los resultados: Se identificaron las debilidades, amenazas, fortalezas y oportunidades que inciden en el fenómeno estudiado, y se diseñó la matriz DAFO de impactos cruzados empleando el procedimiento propuesto por Ronda (2007), se elaboró además con las debilidades el diagrama de Pareto a fin de determinar cuáles clasifican como vitales a partir del principio 80:20. A partir de estos resultados se determinaron las estrategias a desarrollar para revertir la situación actual de la agencia.

Fase 5. Propuesta de acciones de mejora: A partir de los resultados obtenidos y las problemáticas identificadas se definieron las acciones a tener en cuenta para la mejora en la gestión del servicio a cruceristas y al logro de las estrategias.

\section{ANÁLISIS DE RESULTADOS}

Se determinó que existen dos campos de acción con respecto a la rivalidad entre los competidores: la atención a cruceristas y la atención a compañías de cruceros. En cuanto a la atención a cruceristas, la rivalidad entre los competidores se considera alta, debido a que existe una fuerte concentración de estos, el sector privado mediante el uso constante de Internet logra acceder al crucerista con efectividad. Con la reducción de los viajes de norteamericanos a Cuba sin necesidad de utilizar una compañía o grupo, a causa de las nuevas medidas impuestas por la administración de Donald Trump, una buena parte del sector privado ha visto reducida su cuota de mercado e ingresos, lo que ha traído como consecuencia que muchos redirijan sus esfuerzos de venta a los cruceristas, ofertando precios inferiores a los del buque y las agencias estatales.

En cuanto a la atención a las compañías de cruceros el nivel de rivalidad es alto, dado que, aunque el sector privado no tiene participación en esta actividad, existen otras agencias estatales que pugnan por los contratos de atención a las compañías de cruceros, principalmente Havanatur, Cubatur y recientemente Gaviota Tours que se sumó también a esta actividad. Existe en el Grupo Empresarial "Viajes Cuba", al cual se subordinan directamente las agencias de viajes pertenecientes al MINTUR, una orientación hacia una política de continua homogenización de las agencias receptivas, lo que se traduce en una imperceptible diferenciación de sus carteras de producto. Aunque para los cruceros los 
productos tienen ligeras variaciones en los itinerarios o paradas de los programas, los recursos turísticos utilizados son los mismos. Igualmente, los beneficios y niveles de calidad son bastante similares.

En el caso de la agencia Gaviota Tours, la cual se ve beneficiada por el hecho de pertenecer a un grupo en forma de holding, cuenta con prestatarios de la misma compañía, lo que significa menores índices de costos y más facilidades para la negociación, aportándole exclusividad a sus productos. Se evidenció que las barreras de movilidad tanto como las barreras de salida, en el sector de turismo para las agencias de viajes cubanas son muy altas debido a que se subordinan a las políticas de la OSDE Viajes Cuba, por lo tanto, no tienen autonomía suficiente para realizar este tipo de decisiones.

El análisis de la cartera de contratos de la Sucursal Occidente con las compañías de cruceros, evidenció dos líneas bien marcadas de cruceristas atendiendo a las características de los buques: los cruceros de bolsillo o de pequeño porte y los cruceros de mediano porte que atraen el turismo de masas. La primera línea (cruceros de bolsillo) generalmente trae entre 100 y 200 clientes por buque, los cuales se caracterizan por ser de nivel adquisitivo alto y medio alto. Este segmento es generalmente más homogéneo lo que hace más fácil la comercialización y los estudios de mercado (basados en el origen, poder adquisitivo, y otros aspectos). Además, consumen muy bien las excursiones y otros servicios que ofrece la agencia, a pesar de poseer varias opciones ofertadas por la competencia.

En cuanto a la segunda línea de cruceristas, especialmente el segmento que trabaja MSC Cruceros, se caracteriza por su alta heterogeneidad, lo que impide la aplicación de las estrategias de ventas tradicionales, dificulta conocer sus necesidades o hábitos (a pesar de estas condiciones. Se demostró, además, al analizar los documentos operativos de la agencia y al aplicar las encuestas, que los clientes provienen en su mayoría de Europa y Latinoamérica. Suelen ser sensibles a los altos precios de las excursiones una vez a bordo (que por lo general tienen una comisión del 100\%). La tendencia a la conectividad a Internet en los buques permite al cliente investigar y comparar lo que ofrece el crucero con los precios del destino y los actores locales. Además, la terminal Sierra Maestra del puerto de La Habana tiene una característica singular con respecto a las terminales de los puertos de otros países del Caribe, al encontrarse en el centro de la ciudad, facilita al crucerista el encuentro con las ofertas de la competencia, ya que puede explorar por sí mismo y negociar precios directamente con los prestatarios que se encuentran en el área (sector privado). 
Mediante la observación se evidenció que los cruceristas salen de la terminal directamente a explorar por sí mismos las opciones que se ofrecen en el destino, lo que trae consigo que, al poseer tantas opciones, el poder negociador de los cruceristas de MSC Cruceros sea muy elevado. Se realizó una comparación entre el comportamiento de ventas de Celestyal Cruises, compañía, que opera con Cubanacán desde 2015 y que el 90\% de sus pasajeros son estadounidenses, y el comportamiento de MSC Cruceros, que opera en Cuba desde el mismo año y cuyos pasajeros no están sujetos a dichas regulaciones del embargo económico comercial impuesto a la Isla. El buque Crystal (perteneciente a la compañía Celestyal Cruises) por lo general transporta aproximadamente 1200 pasajeros y el de MSC Cruceros entre los 1800 y 2000 pasajeros.

Las encuestas aplicadas a los cruceristas de MSC que visitan el puerto de La Habana, permitieron obtener datos interesantes sobre los hábitos de compra de estos. A continuación, se muestran figuras con los resultados obtenidos en estas encuestas.

\section{Viajes en Crucero}

\section{- Primera vez en crucero - Viajado antes en crucero}

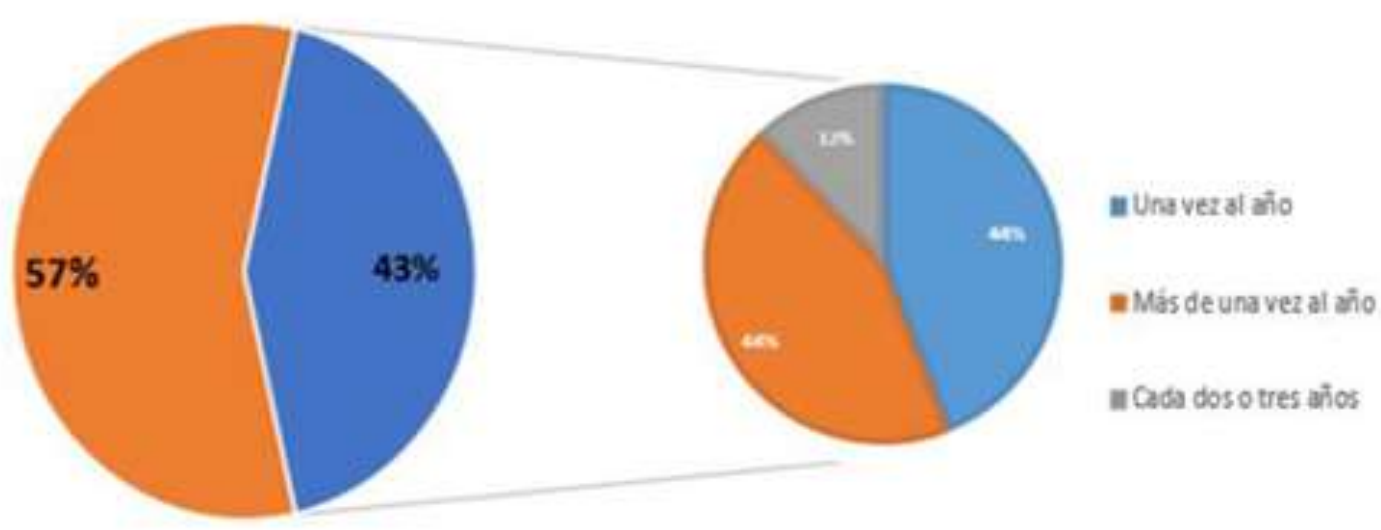

Figura 1: Cantidad de viajes en crucero.

Fuente: Elaboración propia. 


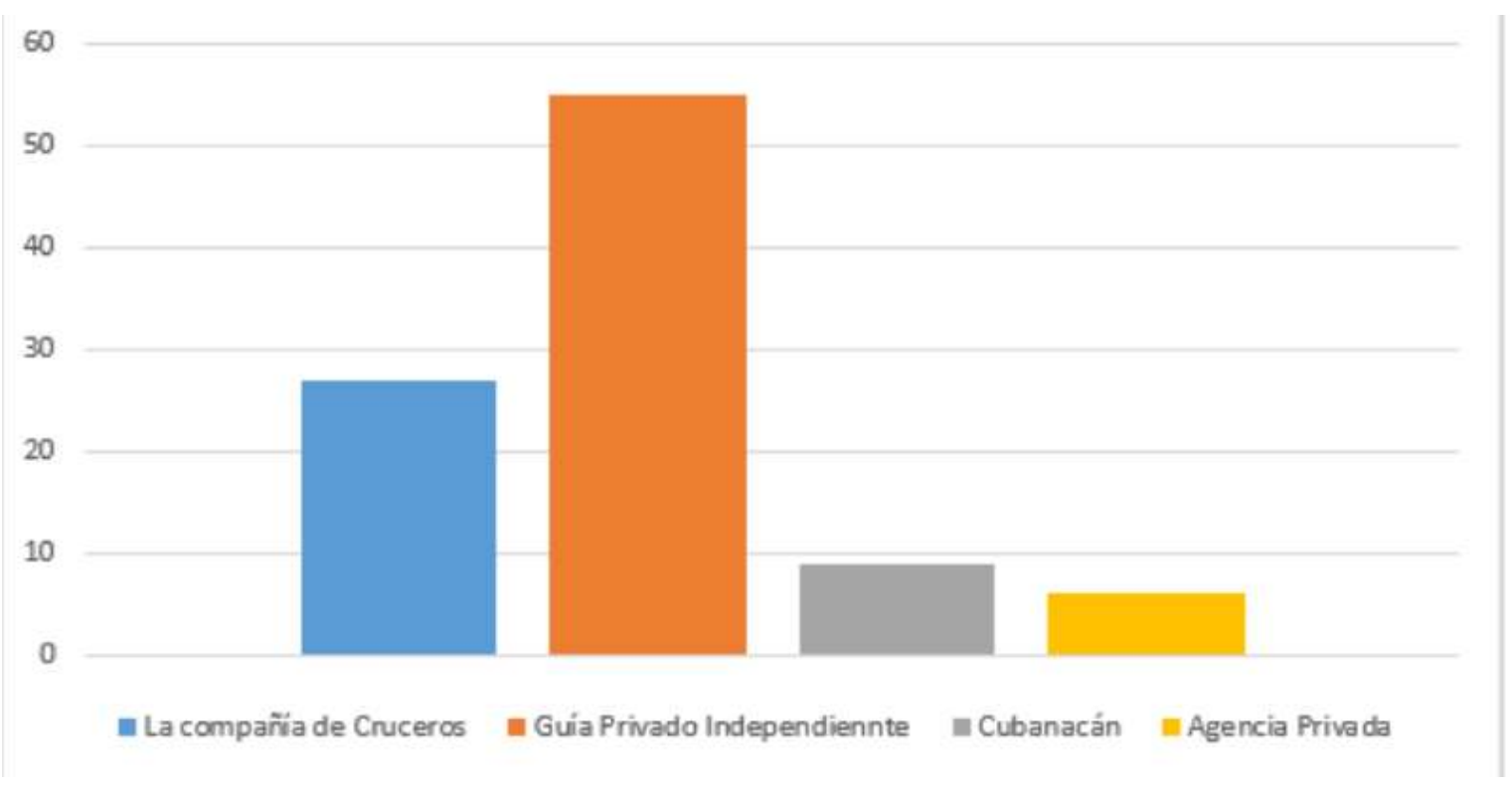

Figura 2: Vías de reservas de excursiones en Cuba.

Fuente: Elaboración propia.

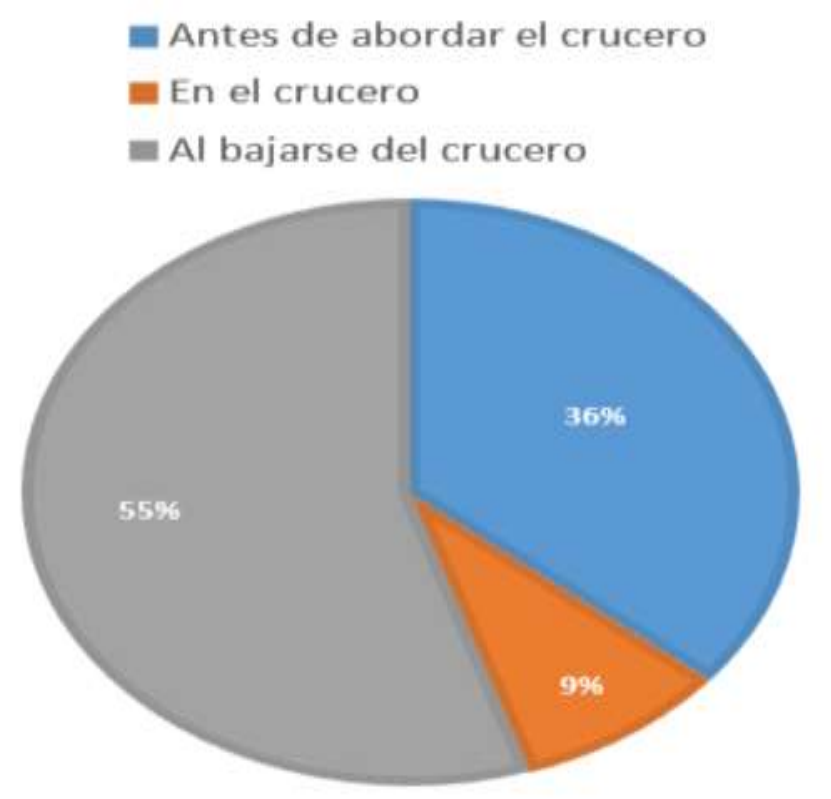

Figura 3: Momento en que se efectúan las reservas de excursiones en Cuba.

Fuente: Elaboración propia. 


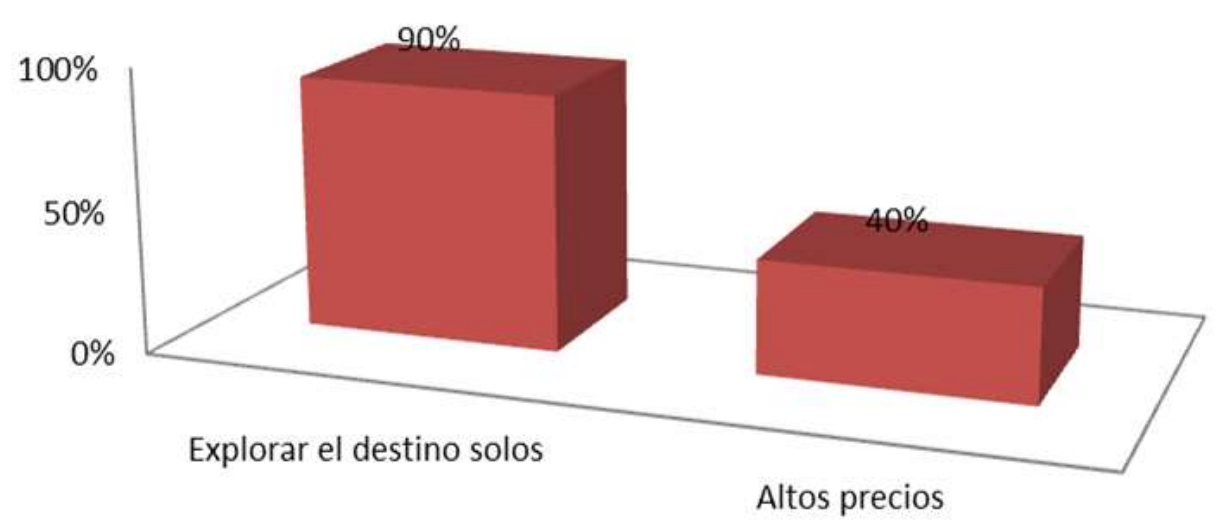

Figura 4: Razones por las que no reservan excursiones en la agencia.

Fuente: Elaboración propia.

\section{Conocen a una agencia de viajes cubana}

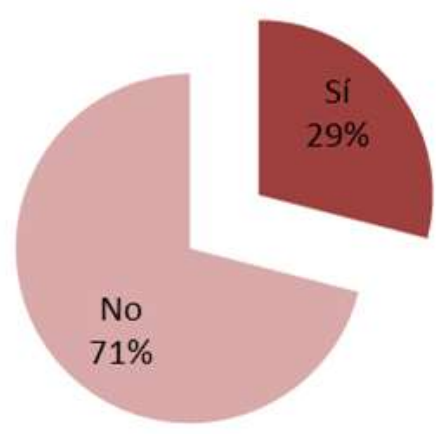

Figura 5: Conocen a una agencia de viajes cubana.

Fuente: Elaboración propia. 


\section{Agencias de viajes conocidas}

\section{ECubatur = Havanatur, Gaviota Tour y Viajes Cubanacán}

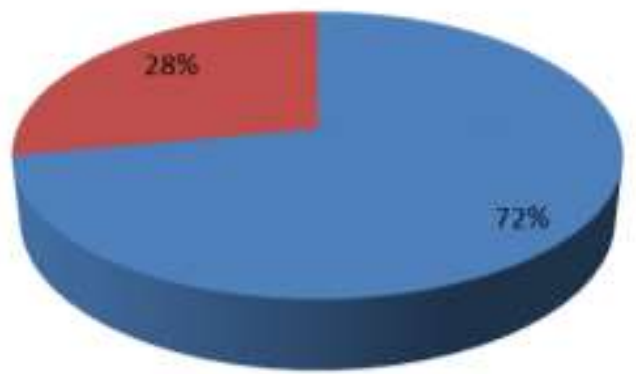

Figura 6: Agencia de viajes cubanas conocidas.

Fuente: Elaboración propia.

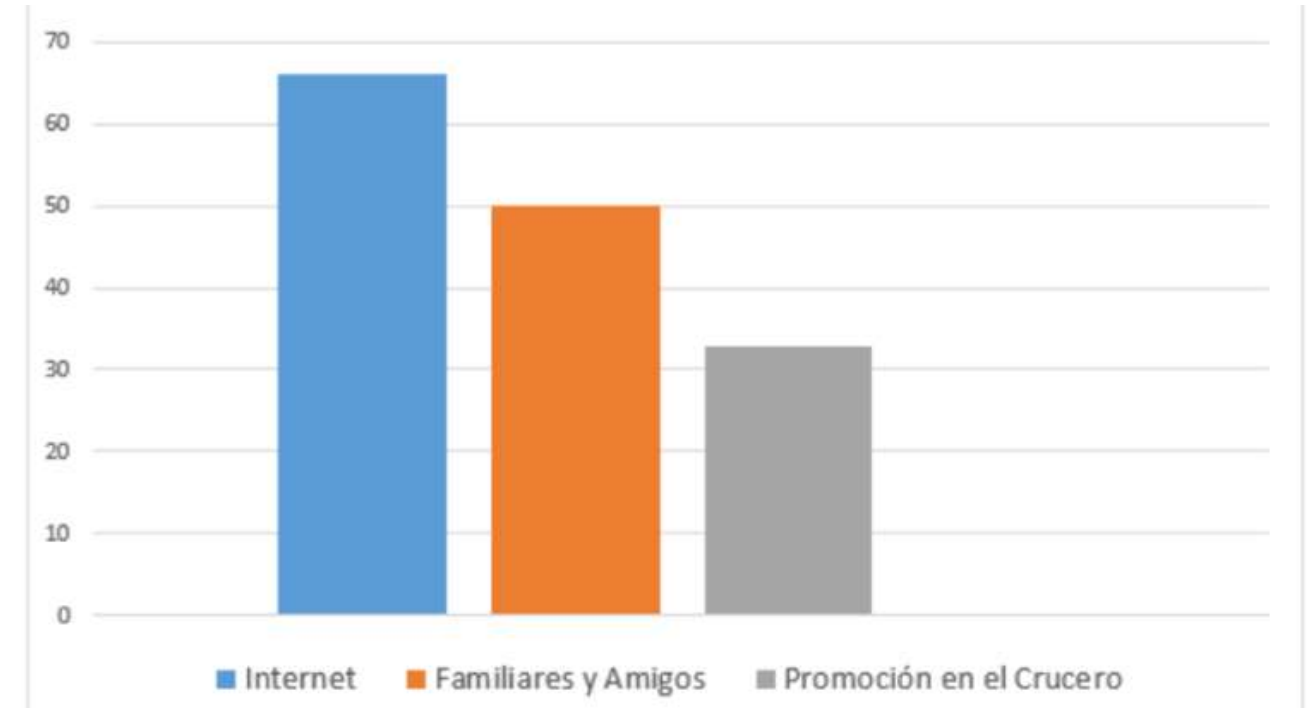

Figura 7: Vías de acceso a la Información de las excursiones del Destino Cuba. Fuente: Elaboración propia.

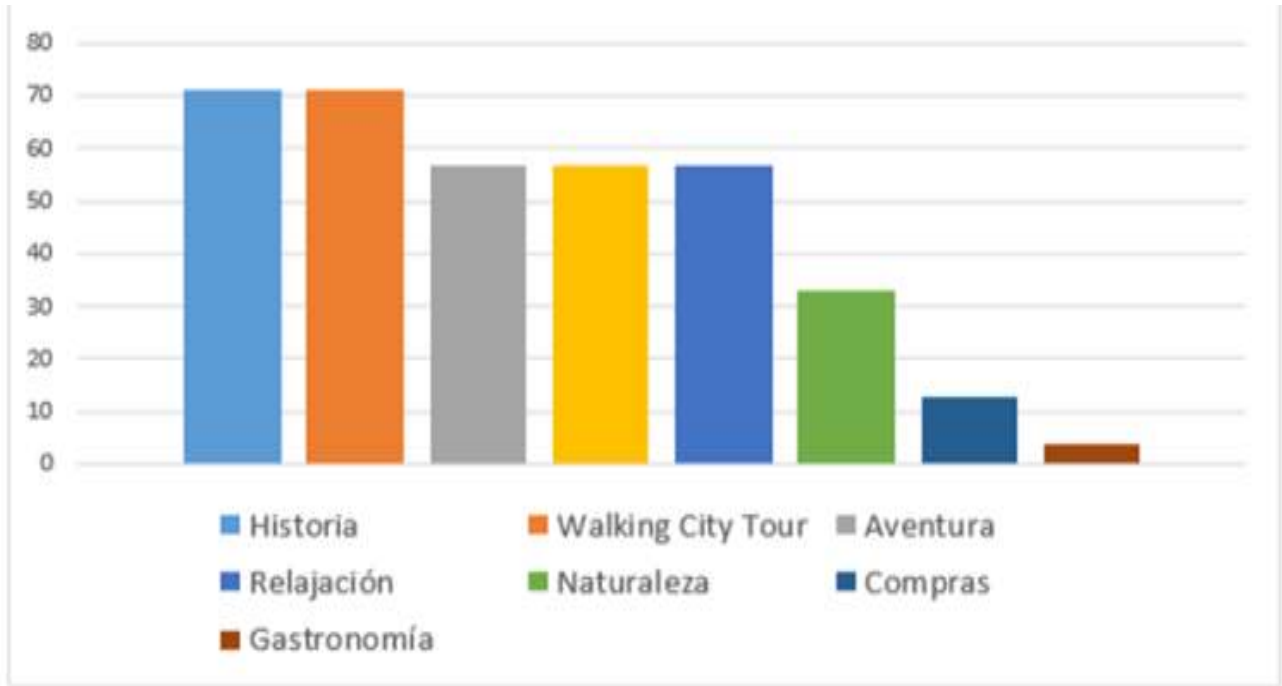

Figura 8: Excursiones preferidas por los cruceristas MSC.

Fuente: Elaboración propia. 
Se determinó que la cartera de productos de la Sucursal Occidente es amplia y diversa, y que, para el diseño de estos se basan principalmente en las experiencias anteriores, disposiciones del Ministerio de Turismo y las oportunidades del destino principalmente. No tienen realizado un estudio de mercado sobre los cruceristas por lo que sus necesidades no se hayan bien identificadas por parte de la agencia. Para los cruceros, la cartera de productos cuenta con 18 excursiones ofertadas a MSC Cruceros, mientras que a Celestyal Cruises oferta 14. Las excursiones más vendidas con dicha compañía en 2017 son en orden descendente: Habana City Tour, City Tour Full Day, Autos Antiguos y Tropicana las cuales presentan márgenes de ganancia por encima de los 80000 CUC anuales.

Se analizó el rendimiento de MSC Cruceros por ser la compañía con la que la agencia presenta bajos niveles de ventas, detectándose que los servicios brindados tienen buena calidad y son bien recibidos por los clientes; que el buen trabajo del guía es referido recurrentemente como elemento diferenciador en el servicio; que existe un desconocimiento del perfil de los clientes por parte de la agencia lo que ha impedido que sus productos, a pesar de poseer calidad, se ajusten completamente a los requerimientos de los cruceristas; la excursión que más se vende por la compañía de cruceros es el City Tour de medio día, cuyo margen de ganancia es del 176\%. Todo ello fue corroborado mediante la revisión de los resultados de las encuestas aplicadas por la agencia a los clientes atendidos durante la temporada 2017-2018, los cuales mostraban un nivel de satisfacción del cliente del $97 \%$. Se determinó que los precios de los productos de la Sucursal Occidente para la compañía de cruceros oscilan entre 23 y 131 cuc, sobre los cuales las líneas de cruceros obtienen un margen de beneficios de un $70 \%$ aproximadamente.

La Sucursal Occidente subordina sus acciones de promoción respecto a los cruceristas a las decisiones de Ventas Internacionales y los armadores de las líneas de cruceros. La utilización de las Tecnologías de la Información y las Comunicaciones es calificada como muy mala. Para la distribución de los productos a los cruceristas utilizan principalmente la Compañía de Cruceros contratada y el correo electrónico (mailing solo para clientes intermedios). En resumen, las formas de promoción y distribución utilizadas no se corresponden con las tendencias actuales de los consumidores de MSC (hecho corroborado en la aplicación de las encuestas).

En relación al personal se evidencia un interés por parte de los trabajadores en la mejora del producto-servicio de la agencia y su adecuación a las tendencias actuales del mercado. No obstante, se debe señalar que no existe un equipo de trabajo especializado en la atención a cruceros, actualmente se encargan de esta labor, dos especialistas comerciales de mercado 
convencional, especialista de calidad, director de operaciones y la coordinadora (sin dejar de lado el resto de las actividades y atención a otros mercados dentro de la Sucursal). No existe personal de venta de la Sucursal a bordo de los buques.

Aun cuando la agencia cuenta con medios informáticos para coordinar y hacer llegar las informaciones necesarias a sus trabajadores, la utilización de estos todavía es restringida y no se aprovechan sus capacidades al máximo. La comunicación para recibir los pedidos del armador se basa fundamentalmente en el correo electrónico y por teléfono. Igualmente sucede con los prestatarios. Para el desarrollo de las operaciones en la terminal la entidad cuenta con una oficina in situ para coordinar cambios de último minuto y responder a cualquier incidencia que pueda ocurrir. Se utilizan radios comunicadores y teléfonos celulares para mantener comunicación constante con los transportistas y las autoridades que regulan las operaciones dentro de la terminal de cruceros. Los principales servicios a cruceros ofrecidos por la Sucursal Occidente son: servicios de Transfer in-out; asistencia en el aeropuerto; venta de visados; excursiones en tierra; asistencia en la terminal de cruceros Sierra Maestra.

Se determinaron como principales Oportunidades: el crucero MSC Armonia a partir de la próxima temporada tendrá su puerto madre en Miami, EEUU; diversificación de los prestatarios con la incorporación del creciente sector privado como oferta turística complementaria; incremento del arribo al puerto de La Habana de importantes compañías de cruceros como Royal Caribbean Cruises, Carnival Cruises, Norwegian Cruise Line, entre otras; la Administración Trump, a pesar de la reversión de muchas de las medidas del gobierno de Barack Obama sobre los viajes a Cuba, excluyó específicamente al turismo de cruceros; aumento del número de cruceristas en el Caribe; y la capacidad de MSC Cruceros de contratar servicios de promoción online inaccesibles para la Sucursal Occidente.

Como principales Amenazas se detectaron la fuerte competencia de precios desde el sector privado; el uso intensivo de Internet y las TICs por parte de la competencia; las decisiones de la OSDE con respecto a la operación de cruceros responden a intereses superiores a los de la Sucursal Occidente; las altas comisiones de las compañías de cruceros sobre los productos de la agencia; la heterogeneidad cada vez mayor de los cruceristas; el aumento de los precios de algunos prestatarios; la ubicación de la Terminal de cruceros de La Habana posibilita el fácil acceso de los clientes a productos sustitutos; la infraestructura insuficiente de la terminal de cruceros para maximizar la eficiencia de la operación; y las restricciones viales dentro y alrededor de la Terminal de cruceros. 
Entre las principales Debilidades detectadas se destacan el bajo poder de negociación de la Sucursal Occidente, con respecto a los contratos; el bajo aprovechamiento de las ventajas de Internet para la promoción y distribución de los productos; la falta de conocimiento por parte de la agencia del perfil de los cruceristas que arriban mediante la compañía MSC Cruceros; la agencia no establece topes de precios para la reventa de sus productos; la agencia no posee personal de venta a bordo de los cruceros ni en la terminal de cruceros; y no existe enfoque de cooperación con el armador para incrementar los niveles de ventas y promoción de los productos de la agencia.

Se determinaron además como principales Fortalezas: el alto interés de los especialistas comerciales en mejorar el comportamiento de las ventas de excursiones a cruceros; la calidad y aceptación entre los clientes de los productos-servicios ofertados por la agencia; la agencia posee experiencia en la atención a cruceristas; la variedad de prestatarios incluyendo el sector privado; y la existencia de documentos guía para la relación contractual y de servicios a los cruceristas.

A partir de la información anterior, se elaboró la matriz DAFO de impactos cruzados, que se presenta en la tabla 1.

Tabla 1. Matriz de Impactos Cruzados.

\begin{tabular}{|c|c|c|c|c|c|c|c|c|c|c|c|c|c|c|c|c|}
\hline & 01 & $\mathrm{O} 2$ & $\mathrm{O} 3$ & $\mathrm{O} 4$ & $\mathrm{O} 5$ & $\mathrm{O} 6$ & $\mathrm{~T}$ & $\mathrm{~A} 1$ & $\mathrm{~A} 2$ & $\mathrm{~A} 3$ & $\mathrm{~A} 4$ & $\mathrm{~A} 5$ & $\mathrm{~A} 6$ & $\mathrm{~A} 7$ & $\mathrm{~A} 8$ & $\mathrm{~T}$ \\
\hline $\mathrm{F} 1$ & 3 & 2 & 2 & 2 & 2 & 3 & 14 & 3 & 2 & 1 & 0 & 3 & 1 & 1 & 1 & 12 \\
\hline F2 & 3 & 0 & 3 & 2 & 1 & 0 & 9 & 3 & 2 & 1 & 1 & 3 & 1 & 2 & 2 & 15 \\
\hline F3 & 2 & 3 & 2 & 2 & 1 & 0 & 10 & 2 & 1 & 3 & 0 & 2 & 1 & 2 & 2 & 13 \\
\hline F4 & 1 & 3 & 2 & 0 & 0 & 2 & 8 & 0 & 0 & 0 & 0 & 0 & 3 & 0 & 1 & 4 \\
\hline F5 & 1 & 0 & 1 & 1 & 3 & 0 & 6 & 3 & 3 & 0 & 3 & 3 & 0 & 3 & 1 & 16 \\
\hline T & 10 & 8 & 10 & 7 & 7 & 5 & 47 & 11 & 8 & 5 & 4 & 11 & 6 & 8 & 7 & 60 \\
\hline D1 & 2 & 2 & 2 & 2 & 2 & 2 & 12 & 3 & 3 & 1 & 3 & 1 & 1 & 0 & 0 & 12 \\
\hline D2 & 3 & 1 & 3 & 3 & 3 & 1 & 14 & 3 & 3 & 0 & 3 & 3 & 0 & 3 & 1 & 16 \\
\hline D3 & 3 & 3 & 3 & 3 & 3 & 2 & 17 & 3 & 3 & 0 & 3 & 3 & 0 & 2 & 1 & 15 \\
\hline D4 & 0 & 0 & 0 & 0 & 0 & 2 & 2 & 3 & 0 & 0 & 3 & 3 & 0 & 3 & 0 & 12 \\
\hline D5 & 1 & 1 & 3 & 3 & 3 & 0 & 11 & 3 & 3 & 0 & 3 & 3 & 0 & 3 & 0 & 15 \\
\hline D6 & 3 & 1 & 0 & 1 & 2 & 3 & 10 & 3 & 3 & 0 & 3 & 2 & 0 & 3 & 0 & 14 \\
\hline T & 12 & 8 & 11 & 12 & 13 & 10 & 66 & 18 & 15 & 1 & 18 & 15 & 1 & 14 & 2 & 84 \\
\hline
\end{tabular}

Fuente: Elaboración propia. 
Posterior a ello se determinó que la agencia se encuentra en el cuadrante de Supervivencia, por lo cual debe desarrollar estrategias dirigidas a minimizar sus debilidades ya que estas contribuyen a una mayor manifestación de las amenazas presentes en el entorno.

Se aplicó además el principio de Pareto encontrándose como principales debilidades la falta de conocimiento por parte de la agencia del perfil de los cruceristas que arriban mediante la compañía MSC Cruceros; el bajo aprovechamiento de las ventajas de Internet para la promoción y distribución de los productos; la agencia no posee personal de venta a bordo de los cruceros ni en la terminal de cruceros y el bajo poder de negociación de la Sucursal Occidente, con respecto a los contratos. Estas debilidades pueden ser minimizadas por la agencia ya que se encuentran en su rango y posibilidades de actuación. En el caso de las amenazas presentes en el entorno, que afectan directamente a la Sucursal Occidente, destacan: la fuerte competencia del sector privado, la heterogeneidad cada vez mayor de los cruceristas y las altas comisiones a los productos de la agencia por parte de las compañías de cruceros.

Se definieron un conjunto de acciones para la Sucursal Occidente en particular y Viajes Cubanacán en general, las cuales se muestran a continuación:

- Realizar estudios de mercado por cada una de las compañías navieras reales y potenciales del destino Cuba.

- Realizar un análisis de benchmarking a los productos que brinda las agencias competidoras de la Sucursal Occidente.

- Aplicar la ingeniería de producto a la cartera de producto de la Sucursal Occidente a los cruceristas.

- Rediseñar la cartera de productos a cruceristas con enfoque a las necesidades reales de los clientes.

- Insertar ofertas de productos, por cada una de las compañías, que se diferencien de los productos ofertados por la competencia.

- Crear los puestos de trabajo de Comunity Manager y Social Manager en la Sucursal Occidente.

- Vincular los perfiles de Facebook e Instagram de la Sucursal Occidente con la página web de viajes Cubanacán.

- Crear un perfil en TripAdvisor que identifique a la agencia Viajes Cubanacán.

- Incluir soportes digitales con acceso a la red en los puntos de venta a cruceristas de la agencia. 
- Realizar negociaciones para incluir personal de ventas de la agencia a bordo de los cruceros.

- Crear un equipo de trabajo especializado, dedicado únicamente en la actividad de los cruceros.

- Establecer precios topes para la reventa, por parte de las compañías, de los productos de la agencia.

\section{CONCLUSIONES}

La industria de cruceros posee un desarrollo vertiginoso, creciendo anualmente el número de cruceristas e ingresos. Las agencias de viajes continúan siendo las intermediarias por excelencia de esta actividad, gestionando aproximadamente el $90 \%$ de la operación. En el destino turístico Cuba, la Sucursal Occidente de la Agencia Viajes Cubanacán, es una de las entidades encargadas de la atención a las compañías de crucero y de los cruceristas.

Los principales factores que afectan a la Sucursal Occidente con respecto a la modalidad turismo de cruceros se centran en el desconocimiento del perfil de los cruceristas, el bajo aprovechamiento de las tecnologías de la información y las comunicaciones, la no existencia de personal de Viajes Cubanacán a bordo de los cruceros, la fuerte competencia del sector privado, la heterogeneidad cada vez mayor de los cruceristas y las altas comisiones a los productos de la agencia por parte de las compañías de cruceros.

Las acciones propuestas están en correspondencia con las problemáticas detectadas y en aras de emplear estrategias de supervivencias; se orientan, fundamentalmente, al aprovechamiento de las fortalezas de la agencia y las oportunidades del entorno, su aplicación contribuirá a revertir la situación actual de la Sucursal Occidente de la Agencia Viajes Cubanacán, donde los ingresos y número de cruceristas se encuentran por debajo de los planes proyectados por la agencia.

\section{REFERENCIAS BIBLIOGRÁFICAS}

Albrecht, K. e. (1991). La gerencia del servicio. Legis.

Association, C. L. ( 2004). The Overview, spring 2004. Retrieved Mayo 5, 2018 from https://msword/reports/overviews/spring04ov.doc.10.dic.2017

Ayala, H. (2014). Agencias de Viajes y Turoperadores en el Turismo. La Habana.

Carlzon, J. (1991). El momento de la verdad. España.: Ed, Díaz de Santos. 
Díaz, L. (2016). Valoración de la Gestión de la Distribución en la Sucursal Occidente de la Agencia de viajes Cubatur S.A. Facultad de Turismo. Universidad de La Habana. Cuba.: En opción al título de Licenciado en Turismo.

Mustelier, D. (2015). Estudio del gasto de los cruceristas que visitan el Puerto de La Habana. Facultad de Turismo. Universidad de La Habana.

Perelló, J. L. (2006). Evolución y Desarrollo de la Industria de Cruceros. Modalidad Turismo de Cruceros. La Habana.: Bibliografía Complementaria Centro de Estudios Turísticos. Universidad de la Habana.

Ulacia, Z. ( 2007). La gestión de Procesos en la Hospitalidad. Universidad de La Habana, La Habana, Cuba.

Ulacia, Z. (2015). Sistemas de Servicio y Hospitalidad. La Habana. Cuba: Félix Varela.

Yi, A. (2018). Propuesta de acciones para incrementar el número de cruceristas e ingresos deseados por la Agencia Viajes Cubanacán Sucursal Occidente. Trabajo de diploma. Facultad de Turismo. Universidad de La Habana. 